\title{
A SOLUTION ALGORITHM FOR $p$-MEDIAN LOCATION PROBLEM ON UNCERTAIN RANDOM NETWORKS
}

\author{
Akram Soltanpour, Fahimeh Baroughi and Behrooz Alizadeh
}

C 2020 by University of Niš, Serbia | Creative Commons License: CC BY-NC-ND

\begin{abstract}
This paper investigates the classical $p$-median location problem in a network in which some of the vertex weights and the distances between vertices are uncertain and while others are random. For solving the $p$-median problem in an uncertain random network, an optimization model based on the chance theory is proposed first and then an algorithm is presented to find the $p$-median. Finally, a numerical example is given to illustrate the efficiency of the proposed method.
\end{abstract}

Keywords: Location problem; p-median; Chance theory; Uncertain random network.

\section{Introduction}

Location problems have received strong theoretical interest due to their relevance in practice. One of the well-known location problems which was considered in the literature is the $p$-median location problem which is stated as follows: Let $N=(V, E)$ be an undirected connected network with vertex set $V,|V|=n$, edge set $E$ and let $p$ be a constant with $p \leq n$. Every edge $e \in E$ has a positive length and each vertex $v_{i} \in V$ is associated with a nonnegative weight $w_{i}$ that it is the demand of the client at this vertex. Let $d(x, y)$ denote the distance between $x, y \in N$ which is equal to the length of the shortest path connecting these two points. In the classical $p$-median problem, the aim is to locate $p$ pairwise different facilities $m_{1}, \ldots, m_{p}$ on the network $N$ (i.e., on vertices or edges) which minimize the sum of weighted distances from each vertex to its closest facility:

$$
\left(\mathbf{P}_{1}\right): \min _{X_{p} \subset N,\left|X_{p}\right|=p} \sum_{v_{i} \in V} w_{i} d\left(v_{i}, X_{p}\right)
$$

where

$$
d\left(v_{i}, X_{p}\right)=\min _{j=1,2, \ldots, p} d\left(v_{i}, m_{j}\right), \quad X_{p}=\left\{m_{1}, \ldots, m_{p}\right\} .
$$

An optimal solution $X_{p}^{*}$ is called a $p$-median.

Received April 19, 2018; accepted August 15, 2019

2010 Mathematics Subject Classification. Primary 90C27; Secondary 90B80 
Hakimi [13] showed that there exists an optimal solution among the set of vertices. This property is called vertex optimality. Later, Kariv and Hakimi [16] proved that the classical $p$-median problem was NP-hard, even if $N$ was a planar graph of maximum degree 3 .

Now, let $d_{i j}=d\left(v_{i}, v_{j}\right)$ be the distance from demand vertex $v_{i}$ to candidate facility at vertex $v_{j}$. Also, let $w=\left\{w_{i} \mid v_{i} \in V\right\}$ and $d=\left\{d_{i j} \mid v_{i}, v_{j} \in V\right\}$ denote the set of the vertex weights and the set of distances between vertices, respectively. Then in the network $N$, the optimal objective value of the $p$-median problem is a function of $w$ and $d$, which is denoted as $f(w, d)$ in this paper.

We are going to present a 0-1 linear programming formulation of the classical discrete $p$-median problem. Let $x_{i j}$ be the variable that is equal to 1 if the demands of the vertex $v_{i}$ are served by a facility at the vertex $v_{j}$, and 0 otherwise. Also, let the variable $x_{j}$ be equal to 1 if there is an open facility at the vertex $v_{j}$, and 0 otherwise. Then, the $0-1$ linear programming formulation of the classical discrete $p$-median problem can be stated as follows:

$$
\begin{array}{rlr}
\left(\mathbf{P}_{2}\right): \min & \sum_{i=1}^{n} \sum_{j=1}^{n} w_{i} d_{i j} x_{i j} & \\
\text { s.t. } & \sum_{j=1}^{n} x_{i j}=1 & \forall i=1, \ldots, n, \\
& x_{i j} \leq x_{j} & \\
& \sum_{j=1}^{n} x_{j}=p, & \\
& x_{i j}, x_{j} \in\{0,1\} & \forall i, j=1, \ldots, n,
\end{array}
$$

This model minimizes the total weighted distance between each demand vertex and the nearest facility. The first set of constraints requires each demand vertex to be assigned to exactly one facility. The second set of constraints allows the demand of the vertex $v_{i}$ to be assigned to a vertex $v_{j}$ only if there is an open facility in this location. The third set of constraints states that exactly $p$ facilities are to be located. Finally, the last constraints are the standard integrality conditions [8, 28].

The $p$-median location problems have been studied by many researchers. Kariv and Hakimi [16] presented an $O\left(p^{2} n^{2}\right)$ time algorithm for the $p$-median location problem on tree networks. Gavish and Sridhar [10] proposed an algorithm for the 2-median problem on trees which is run in $O(n \log n)$ time. Tamir [36] improved the time complexity of the $p$-median problem on trees to $O\left(p n^{2}\right)$. Benkoczi and Bhattacharya [6] presented an algorithm with time complexity of $O(p n \log n)$ for solving the $p$-median problem on interval networks. Also, they designed an $O\left(n \log ^{p+2} n\right)$ time algorithm to solve the $p$-median problem on trees [1]. For the 1-median problem on wheel networks, Hatzl [14] provided an algorithm with linear running time. In addition, he showed that the 2-median problem on the cactus networks can be calculated at $O\left(n^{2}\right)$ time. Chang et al. [7] proved that the connected $p$-median problem on block graphs was $\mathcal{N} \mathcal{P}$-hard and for the case that the lengths of the edges were equal to one, he proposed an $O(n)$ time algorithm. In 2017, Nguyen et 
al. [30]presented a simple algorithm with linear running time to find the 1-median location on a cactus network. In the real life, we are usually faced with various types of non-determinacy. For example, in location problems, we are usually not sure of the vertex weights and the distances between vertices of a network. For dealing with non-determinacy phenomena, probability theory was introduced by Kolmogorov [17] in 1933 for modeling frequencies, while in 2007, uncertainty theory was presented by Liu [18] for modeling belief degrees.

The early works mainly focus on handling randomness, i.e., regarding distances between vertices and vertex weights as random variables. Berman and Krass [5] investigated the $p$-median problem with unreliable facilities and complete information on a line. They presented an approach for solving the problem that was based on representing the stochastic problem as a linear combination of deterministic median problems for which analytical results are available. Berman and Wang [3] studied the problem of locating $p$ facilities to serve clients residing at the vertices of a network with discrete probabilistic demand weights. Tadei et al. [37] tried to find the location of $p$ facilities when the cost for using a facility is a stochastic variable with unknown probability distribution. Berman and Drezner [4] investigated the $p$-median problem with stochastic objective value. Their aim was to find the location of $p$ facilities such that the expected value of the objective function in the future is minimized. When the stochastic network is a tree, Mirchandani and Oudjit [27] used a selective enumeration approach for solving the 2-median problem. Various results on the $p$-median problem on stochastic networks are given in $[2,9,23,24,25,26]$.

Stochastic network optimization models work well when there are enough data to estimate the probability distributions of vertex weights and distances between the vertices. When we do not have enough samples to estimate the probability distributions of the vertex weights and the distances between vertices, we have to invite experts to give the belief degrees about the vertex weights and distances between vertices. Some researchers applied the uncertainty theory to deal with the location problems: for example the uncertain models for single facility location problems were investigated by Yuan Gao [12], the hierarchical facility location for the reverse logistics network design under uncertainty was studied by Wang and Yang [38], the capacitated facility location-allocation problem under uncertain environment was investigated by Wen et al. [39], the inverse 1-median problem on a tree under uncertain cost coefficients was solved by Nguyen and Chi [29] and the classical $p$-center location problem on a network with the uncertain vertex weights and the uncertain distances was studied by Soltanpour et al. [35].

In many cases, uncertainty and randomness simultaneously appear in a complex system. Specifically, for some non-deterministic phenomena, we have enough observational data to obtain their probability distribution functions, while for others, we can only estimate them by expert data. In order to describe this complex system, in 2013 the chance theory was developed by Liu [21] with the concepts of uncertain random variable, chance measure and chance distribution. Liu [21] also introduced the concepts of expected value and variance of uncertain random variables. For cal- 
culating the variance of uncertain random variables, Guo and Wang [11] presented a formula based on uncertainty distribution. Sheng and Yao [34] verified a formula to calculate the variance using chance distribution and inverse chance distribution. As an important contribution to chance theory, Liu [22] presented an operational law of uncertain random variables. In addition, Hou [15] investigated the distance between uncertain random variables, and Yao and Gao [40] proved a law of large numbers for the uncertain random variables. In order to model the optimization problems with uncertainty and randomness, uncertain random programming was introduced by Liu [22] in 2013. For a survey on the uncertain random optimization problems, the reader is referred to $[31,32,33]$.

In this paper, we will investigate the $p$-median location problem on a network with uncertain random vertex weights and distances. In the $p$-median problem too, there are some circumstances by which there are enough data for some of vertex weights and distances to estimate their probability distributions. On the other hand, there are no samples to estimate the probability distributions for some other vertex weights and distances so that we have to invite experts to give the belief degrees about them. Therefore, data are divided into categories; some of them have probability distributions and the others have uncertainty distributions.

The article is organized as follows: In the next section some basic concepts and properties of the uncertainty theory and chance theory will be introduced. In Section 3., we will introduce an uncertain random network and give an ideal chance distribution function of the $p$-median. Then, we propose an algorithm to calculate the ideal chance distribution function of the problem under investigation on uncertain random networks. Section 4 . presents a model for finding the $p$-median and proposes an algorithm to seek the $p$-median of an uncertain random network. A numerical example is presented in Section 5. to illustrate the efficiency of our proposed method. Finally, Section 6 . gives a brief summary to the paper.

\section{Preliminary concepts and definitions}

In this section, we will introduce some concepts and theorems of the uncertainty theory and chance theory.

\subsection{Uncertainty theory}

The uncertainty theory, introduced by Liu [18], provides a new approach to deal with non-determinacy factors. Nowadays, the uncertainty theory has become a branch of mathematics for modeling human uncertainty based on normality, duality, subadditivity, and product axioms.

In the following part, we will introduce some foundational concepts and properties of the uncertainty theory, which will be used throughout this paper $[18,19,20]$.

Definition 2.1. Let $\Gamma$ be a nonempty set, $\mathcal{L}$ a $\sigma$-algebra over $\Gamma$. A set function $\mathcal{M}: \mathcal{L} \rightarrow[0,1]$ is called an uncertain measure if it satisfies the following axioms: 
Axiom 1: (Normality Axiom) $\mathcal{M}\{\Gamma\}=1$ for the universal set $\Gamma$.

Axiom 2: ( Duality Axiom) $\mathcal{M}\{\Lambda\}+\mathcal{M}\left\{\Lambda^{c}\right\}=1$ for any event $\Lambda \in L$ ( $\Lambda^{c}$ is compliment of $\Lambda)$.

Axiom 3: (Subadditivity Axiom) For every countable sequence of events $\Lambda_{1}, \Lambda_{2}, \ldots$, we have

$$
\mathcal{M}\left\{\bigcup_{i=1}^{\infty} \Lambda_{i}\right\} \leq \sum_{i=1}^{\infty} \mathcal{M}\left\{\Lambda_{i}\right\} .
$$

The triple $(\Gamma, \mathcal{L}, \mathcal{M})$ is called an uncertainty space. Moreover, in order to provide an operational law, Liu defined the product uncertain measure on the product $\sigma$-algebra $\mathrm{L}$ as follows.

Axiom 4: (Product Axiom) Let $\left(\Gamma_{k}, \mathcal{L}_{k}, \mathcal{M}_{k}\right)$ be uncertainty spaces for $k=1,2, \ldots$. Then the product uncertain measure $\mathcal{M}$ is an uncertain measure satisfying

$$
\mathcal{M}\left\{\prod_{k=1}^{\infty} \Lambda_{k}\right\}=\bigwedge_{k=1}^{\infty} \mathcal{M}\left\{\Lambda_{k}\right\}
$$

where $\Lambda_{k}$ are arbitrary chosen events from $\mathcal{L}_{k}$ for $k=1,2, \ldots$, respectively.

Definition 2.2. An uncertain variable is a measurable function $\xi$ from an uncertainty space to the set of real numbers, i.e., for any Borel set B of real numbers, the set

$$
\{\xi \in B\}=\{\gamma \in \Gamma \mid \xi(\gamma) \in B\}
$$

is an event.

In order to describe an uncertain variable, a concept of uncertainty distribution is defined as follows.

Definition 2.3. The uncertainty distribution function $\phi$ of an uncertain variable $\xi$ is defined by

$$
\phi(x)=\mathcal{M}\{\xi \leq x\}
$$

for any real number $x$.

Definition 2.4. The uncertain variables $\xi_{1}, \xi_{2}, \ldots, \xi_{n}$ are said to be independent if

$$
\mathcal{M}\left\{\bigcap_{i=1}^{n}\left\{\xi_{i} \in B_{i}\right\}\right\}=\bigwedge_{i=1}^{n} \mathcal{M}\left\{\xi_{i} \in B_{i}\right\}
$$

for any Borel sets $B_{1}, B_{2}, \ldots, B_{n}$ of real numbers.

Definition 2.5. Let $\xi$ be an uncertain variable with regular uncertainty distribution function $\phi$. Then the inverse function $\phi^{-1}$ is called the inverse uncertainty distribution function of $\xi$. 
The distribution of a monotonous function of uncertain variables can be obtained by the following theorem.

Theorem 2.1. Let $\xi_{1}, \xi_{2}, \ldots, \xi_{n}$ be the independent uncertain variables with regular uncertainty distribution functions $\phi_{1}, \phi_{2}, \ldots, \phi_{n}$, respectively. If the function $f\left(x_{1}, x_{2}, \ldots, x_{n}\right)$ is strictly increasing with respect to $x_{1}, x_{2}, \ldots, x_{n}$, then $\nu=f\left(\xi_{1}, \xi_{2}, \ldots, \xi_{n}\right)$ is an uncertain variable with inverse uncertainty distribution function

$$
\psi^{-1}(\alpha)=f\left(\phi_{1}^{-1}(\alpha), \phi_{2}^{-1}(\alpha), \ldots, \phi_{n}^{-1}(\alpha)\right) .
$$

\subsection{Chance theory}

In this subsection, we will introduce some foundational definitions and properties of the uncertain random variable, the chance measure, the chance distribution and the operational law, [21, 22].

Let $(\Gamma, \mathcal{L}, \mathcal{M})$ be an uncertainty space and $(\Omega, \mathcal{A}, \operatorname{Pr})$ be a probability space. The product $(\Gamma, \mathcal{L}, \mathcal{M}) \times(\Omega, \mathcal{A}, \operatorname{Pr})$ is called a chance space.

Definition 2.6. Let $(\Gamma, \mathcal{L}, \mathcal{M}) \times(\Omega, \mathcal{A}, \operatorname{Pr})$ be a chance space, and let $\Theta \in \mathcal{L} \times \mathcal{A}$ be an uncertain random event. Then the chance measure of $\Theta$ is defined as

$$
C h\{\Theta\}=\int_{0}^{1} \operatorname{Pr}\{\omega \in \Omega \mid \mathcal{M}\{\gamma \in \Gamma \mid(\gamma, \omega) \in \Theta\} \geq r\} d r
$$

Liu [21] proved that a chance measure satisfies normality, duality, and monotonicity properties, that is

(1) $C h\{\Gamma \times \Omega\}=1$.

(2) $C h\{\Theta\}+C h\left\{\Theta^{c}\right\}=1$ for any event $\Theta\left(\Theta^{c}\right.$ is compliment of $\left.\Theta\right)$.

(3) $C h\left\{\Theta_{1}\right\} \leq C h\left\{\Theta_{2}\right\}$ for any real number set $\Theta_{1} \subset \Theta_{2}$.

Moreover, Hou [15] proved the subadditivity of chance measure, that is,

$$
C h\left\{\bigcup_{i=1}^{\infty} \Theta_{i}\right\} \leq \sum_{i=1}^{\infty} \operatorname{Ch}\left\{\Theta_{i}\right\}
$$

for a sequence of events $\Theta_{1}, \Theta_{2}, \ldots$.

Theoretically, an uncertain random variable is a measurable function on the chance space. It is usually used to deal with measurable functions of uncertain variables and random variables.

Definition 2.7. An uncertain random variable is a measurable function $\theta$ from a chance space $(\Gamma, \mathcal{L}, \mathcal{M}) \times(\Omega, \mathcal{A}, P r)$ to the set of real numbers, i.e., $\{\theta \in B\}$ is an event for any Borel set $B$. 
Note that random variables and uncertain variables can be regarded as special cases of uncertain random variables.

Definition 2.8. The chance distribution function of an uncertain random variable $\theta$ is defined by

$$
\Phi(x)=C h\{\theta \leq x\}
$$

for any real number $x$.

The chance distribution function of a random variable is just its probability distribution function, and the chance distribution function of an uncertain variable is just its uncertainty distribution function.

Theorem 2.2. Let $\eta_{1}, \eta_{2}, \ldots, \eta_{m}$ be the independent random variables with probability distribution functions $\Psi_{1}, \Psi_{2}, \ldots, \Psi_{m}$ respectively, and let $\tau_{1}, \tau_{2}, \ldots, \tau_{n}$ be the uncertain variables. Then the uncertain random variable

$$
\theta=f\left(\eta_{1}, \eta_{2}, \ldots, \eta_{m}, \tau_{1}, \tau_{2}, \ldots, \tau_{n}\right)
$$

has a chance distribution function

$$
\Phi(x)=\int_{\mathcal{R}^{m}} F\left(x, y_{1}, y_{2}, \ldots, y_{m}\right) d \Psi_{1}\left(y_{1}\right) d \Psi_{2}\left(y_{2}\right) \ldots d \Psi_{m}\left(y_{m}\right)
$$

where $F\left(x, y_{1}, y_{2}, \ldots, y_{m}\right)$ is the uncertainty distribution function of uncertain variable $f\left(y_{1}, y_{2}, \ldots, y_{m}, \tau_{1}, \tau_{2}, \ldots, \tau_{n}\right)$ for any real numbers $y_{1}, y_{2}, \ldots, y_{m}$.

\section{The ideal chance distribution function of the $p$-median}

In this section, we will introduce the uncertain random network and the ideal chance distribution function of $p$-median. Then, we propose an algorithm for calculating the ideal chance distribution function. First, some assumptions are listed as follows.

(1) The undirected uncertain random network is connected.

(2) The weight of each vertex and the distances (shortest path length) between vertices are finite.

(3) The weight of each vertex and the distances between vertices are positive uncertain variables or positive random variables.

(4) All the uncertain variables and the random variables are independent.

Definition 3.1. The quartette $(\mathcal{V}, \mathcal{U}, \mathcal{R}, \mathcal{W})$ is said to be an uncertain random network if $\mathcal{V}=\left\{v_{1}, v_{2}, \ldots, v_{n}\right\}$ is the vertex set and $\mathcal{U}, \mathcal{R}, \mathcal{W}$ are defined as follows.

$\mathcal{U}=\left\{\left(v_{i}, v_{j}\right) \mid\right.$ the shortest distance between vertices $v_{i}$ and $v_{j}$ is uncertain $\}$

$$
\bigcup\left\{v_{k} \mid \text { the weight of the vertex } v_{k} \text { is uncertain }\right\},
$$


$\mathcal{R}=\left\{\left(v_{i}, v_{j}\right) \mid\right.$ the shortest distance between vertices $v_{i}$ and $v_{j}$ is random $\}$

$$
\bigcup\left\{v_{k} \mid \text { the weight of thevertex } v_{k} \text { is random }\right\} \text {, }
$$

and $\mathcal{W}$ is the collection of uncertain and random vertex weights and uncertain and random vertex distances.

In this paper, all deterministic distances and weights are regarded as special uncertain distances and weights.

Let $\eta_{k}$ and $\xi_{i j}$ denote the weight of vertex $v_{k}, v_{k} \in \mathcal{U} \cup \mathcal{R}$, and the distance between two vertices $v_{i}$ and $v_{j},\left(v_{i}, v_{j}\right) \in \mathcal{U} \cup \mathcal{R}$, respectively. Then $\eta_{k}$ and $\xi_{i j}$ are uncertain variables if $v_{k},\left(v_{i}, v_{j}\right) \in \mathcal{U}$, and $\eta_{k}$ and $\xi_{i j}$ are random variables if $v_{k},\left(v_{i}, v_{j}\right) \in \mathcal{R}$. Without loss of generality, we assume that the uncertain weight $\eta_{k}$ and the distance $\xi_{i j}$ for $v_{k},\left(v_{i}, v_{j}\right) \in \mathcal{U}$ are defined on uncertainty spaces $\left(\Gamma_{1}, \mathcal{L}_{1}, \mathcal{M}_{1}\right)$ and $\left(\Gamma_{2}, \mathcal{L}_{2}, \mathcal{M}_{2}\right)$, respectively, and also the random weight $\eta_{k}$ and the distance $\xi_{i j}$ for $v_{k},\left(v_{i}, v_{j}\right) \in \mathcal{R}$ are defined on probability spaces $\left(\Omega_{1}, \mathcal{A}_{1}, P r_{1}\right)$ and $\left(\Omega_{2}, \mathcal{A}_{2}, P r_{2}\right)$, respectively. Since the weights and the distances are assumed to be finite, we have $a_{k} \leq \eta_{k} \leq b_{k}, a_{i j} \leq \xi_{i j} \leq b_{i j}$, where $a_{k}, a_{i j}$ and $b_{k}, b_{i j}$ are the lower bounds and the upper bounds, respectively.

Define $\eta=\left\{\eta_{k} \mid v_{k} \in \mathcal{U} \cup \mathcal{R}\right\}$ and $\xi=\left\{\xi_{i j} \mid\left(v_{i}, v_{j}\right) \in \mathcal{U} \cup \mathcal{R}\right\}$. We can denote the network with the uncertain random weights and distances as $(\mathcal{V}, \mathcal{U}, \mathcal{R}, \mathcal{W})$. The optimal value of the $p$-median problem is a function of weights and distances which is denoted as $f$ in this paper. Obviously, $f(\eta, \xi)$ is an uncertain random variable. For an uncertain random network, the optimal value of the $p$-median problem, $f(\eta, \xi)$, is an increasing function with respect to each component of $\eta_{k}$ and $\xi_{i j}$. The chance distribution function of $f(\eta, \xi)$ is called an ideal chance distribution function associated with uncertain random network $(\mathcal{V}, \mathcal{U}, \mathcal{R}, \mathcal{W})$. Note that the ideal chance distribution function is unique for a given uncertain random network. The following theorem explains how to calculate an ideal chance distribution function.

Theorem 3.1. Let $(\mathcal{V}, \mathcal{U}, \mathcal{R}, \mathcal{W})$ be an uncertain random network. Assume that the uncertain weights $\eta_{k}$ and the uncertain distances $\xi_{i j}$ have regular uncertainty distribution functions $\Upsilon_{k}$ and $\Upsilon_{i j}$ for $v_{k} \in \mathcal{U}$ and $\left(v_{i}, v_{j}\right) \in \mathcal{U}$ and also the random weights $\eta_{k}$ and the random distances $\xi_{i j}$ have probability distribution functions $\Psi_{k}$ and $\Psi_{i j}$ for $v_{k} \in \mathcal{R}$ and $\left(v_{i}, v_{j}\right) \in \mathcal{R}$, respectively. Then the ideal chance distribution function associated with the uncertain random network $(\mathcal{V}, \mathcal{U}, \mathcal{R}, \mathcal{W})$ is

$$
\begin{array}{r}
\Phi(z)=\quad \int_{0}^{\infty} \ldots \int_{0}^{\infty} F\left(z ; y_{k}, y_{i j} \mid v_{k},\left(v_{i}, v_{j}\right) \in \mathcal{R}\right) \\
\prod_{v_{k} \in \mathcal{R}} d \Psi_{k}\left(y_{k}\right) \prod_{\left(v_{i}, v_{j}\right) \in \mathcal{R}} d \Psi_{i j}\left(y_{i j}\right)
\end{array}
$$

where $F\left(z ; y_{k}, y_{i j} \mid v_{k},\left(v_{i}, v_{j}\right) \in \mathcal{R}\right)$ is the uncertainty distribution function of the uncertain variable $f\left(y_{k}, y_{i j}\left|v_{k},\left(v_{i}, v_{j}\right) \in \mathcal{R} ; \eta_{k}, \xi_{i j}\right| v_{k},\left(v_{i}, v_{j}\right) \in \mathcal{U}\right)$, and it is determined by its inverse uncertainty distribution function $F^{-1}\left(\alpha ; y_{k}, y_{i j} \mid v_{k},\left(v_{i}, v_{j}\right) \in \mathcal{R}\right)$ which is equal to

$$
f\left(y_{k}, y_{i j}\left|v_{k},\left(v_{i}, v_{j}\right) \in \mathcal{R} ; \Upsilon_{k}^{-1}(\alpha), \Upsilon_{i j}^{-1}(\alpha)\right| v_{k},\left(v_{i}, v_{j}\right) \in \mathcal{U}\right)
$$

and $f$ can be calculated by using $\left(\mathbf{P}_{2}\right)$. 
Proof. Let $f\left(\eta_{k}, \xi_{i j} \mid v_{k},\left(v_{i}, v_{j}\right) \in \mathcal{U} \cup \mathcal{R}\right)$ be the sum of the weighted distance of the $p$-median. By Definitions 2.6 and 2.8 and also Theorem 2.2, we can obtain the ideal chance distribution function as follows.

$$
\begin{aligned}
\Phi(z)= & \operatorname{Ch}\left\{f\left(\eta_{k}, \xi_{i j} \mid v_{k},\left(v_{i}, v_{j}\right) \in \mathcal{U} \cup \mathcal{R}\right) \leq z\right\} \\
= & \int_{0}^{1} \operatorname{Pr}\left\{\omega_{1} \in \Omega_{1}, \omega_{2} \in \Omega_{2} \mid \mathcal{M}\left\{f \left(\eta_{k}\left(\omega_{1}\right), \xi_{i j}\left(\omega_{2}\right) \mid v_{k},\left(v_{i}, v_{j}\right) \in \mathcal{R},\right.\right.\right. \\
& \left.\left.\left.\eta_{k}, \xi_{i j} \mid v_{k},\left(v_{i}, v_{j}\right) \in \mathcal{U}\right) \leq z\right\} \geq r\right\} d r \\
= & \int_{0}^{\infty} \ldots \int_{0}^{\infty} \mathcal{M}\left\{f\left(\eta_{k}\left(\omega_{1}\right), \xi_{i j}\left(\omega_{2}\right)\left|v_{k},\left(v_{i}, v_{j}\right) \in \mathcal{R}, \eta_{k}, \xi_{i j}\right| v_{k},\left(v_{i}, v_{j}\right) \in \mathcal{U}\right) \leq z\right\} \\
& \quad \prod_{v_{k} \in \mathcal{R}} d \Psi_{k}\left(y_{k}\right) \prod_{\left(v_{i}, v_{j}\right) \in \mathcal{R}} d \Psi_{i j}\left(y_{i j}\right) \\
= & \int_{0}^{\infty} \ldots \int_{0}^{\infty} F\left(z ; y_{k}, y_{i j} \mid v_{k},\left(v_{i}, v_{j}\right) \in \mathcal{R}\right) \prod_{v_{k} \in \mathcal{R}} d \Psi_{k}\left(y_{k}\right) \prod_{\left(v_{i}, v_{j}\right) \in \mathcal{R}} d \Psi_{i j}\left(y_{i j}\right)
\end{aligned}
$$

where $F\left(z ; y_{k}, y_{i j} \mid v_{k},\left(v_{i}, v_{j}\right) \in \mathcal{R}\right)$ is the uncertainty distribution function of uncertain variable $f\left(y_{k}, y_{i j}\left|v_{k},\left(v_{i}, v_{j}\right) \in \mathcal{R} ; \eta_{k}, \xi_{i j}\right| v_{k},\left(v_{i}, v_{j}\right) \in \mathcal{U}\right)$ for any real numbers $y_{k}, y_{i j}, v_{k},\left(v_{i}, v_{j}\right) \in \mathcal{R}$, and it is determined by its inverse uncertainty distribution $F^{-1}\left(\alpha ; y_{k}, y_{i j}, v_{k},\left(v_{i}, v_{j}\right) \in \mathcal{R}\right)$. By Theorem 2.1, for given $\alpha \in(0,1)$, we have

$$
\begin{aligned}
& F^{-1}\left(\alpha ; y_{k}, y_{i j}, v_{k},\left(v_{i}, v_{j}\right) \in \mathcal{R}\right) \\
& =f\left(y_{k}, y_{i j}\left|v_{k},\left(v_{i}, v_{j}\right) \in \mathcal{R} ; \Upsilon_{k}^{-1}(\alpha), \Upsilon_{i j}^{-1}(\alpha)\right| v_{k},\left(v_{i}, v_{j}\right) \in \mathcal{U}\right),
\end{aligned}
$$

which is just the optimal value of the $p$-median problem of a determinacy network and $f$ is a strictly increasing function with respect to $\eta_{k}$ and $\xi_{i j}$, where $\eta_{k}$ and $\xi_{i j}$ denote the weight of the vertex $v_{i}$ and the shortest distance between two vertices $v_{i}$ and $v_{j}$, respectively. We can calculate $f$ by using $\left(\mathbf{P}_{2}\right)$. Thus the theorem is proved.

Note that, it is difficult to calculate the ideal chance distribution function by using formula (3.1). Hence, in order to calculate the ideal chance distribution function of the $p$-median in an uncertain random network, we propose the following algorithm:

\section{Algorithm 1}

1. For any $y_{k}$ and $y_{i j}, v_{k},\left(v_{i}, v_{j}\right) \in \mathcal{R}$, give partitions $\prod_{k}$ and $\prod_{i j}$ of intervals $\left[a_{k}, b_{k}\right]$ and $\left[a_{i j}, b_{i j}\right]$ with step $\Delta=0.01$. Let random variables $\eta_{k}$ and $\xi_{i j}$ take values in $\left\{y_{k} \mid y_{k}=a_{k}+0.01 * i\right.$ for $\left.i=1,2, \ldots,\left(b_{k}-a_{k}\right) * 100\right\}$ and $\left\{y_{i j} \mid y_{i j}=a_{i j}+0.01 * i\right.$ for $\left.i=1,2, \ldots,\left(b_{i j}-a_{i j}\right) * 100\right\}$, respectively.

2. Calculate $F^{-1}\left(\alpha ; y_{k}, y_{i j}, v_{k},\left(v_{i}, v_{j}\right) \in \mathcal{R}\right)$ by using $\left(\mathbf{P}_{2}\right)$ for given $y_{k}$ and $y_{i j}$ and each $\alpha \in\{0.01,0.02, \ldots, 0.99\}$.

3. Obtain the uncertainty distribution function of $F\left(z ; y_{k}, y_{i j} \mid v_{k},\left(v_{i}, v_{j}\right) \in \mathcal{R}\right)$, from its discrete form via linear interpolation.

4. Input $F\left(z ; y_{k}, y_{i j} \mid v_{k},\left(v_{i}, v_{j}\right) \in \mathcal{R}\right)$ into formula (3.1) to calculate the chance distribution function $\Phi(z)$ for each $z$. 


\section{The $p$-median model in uncertain random networks}

In this section, we will consider the $p$-median problem in an uncertain random network and present an algorithm for finding the $p$-median.

Given a connected and undirected uncertain random network $(\mathcal{V}, \mathcal{U}, \mathcal{R}, \mathcal{W})$. Let $\mathcal{V}_{p} \subseteq \mathcal{V},\left|\mathcal{V}_{p}\right|=p$, and let the variable $x_{j}$ be equal to 1 if $v_{j} \in \mathcal{V}_{p}$, and 0 otherwise. Also let $x_{i j}$ be the variable that is equal to 1 if the nearest vertex to the vertex $v_{i}$ in $\mathcal{V}_{p}$ is the vertex $v_{j}$, and 0 otherwise. A $p$-facility location is represented by $\left\{x_{j}, x_{i j} \mid v_{j},\left(v_{i}, v_{j}\right) \in \mathcal{U} \cup \mathcal{R}\right\}$, where

$$
\begin{array}{ll}
\sum_{\left(v_{i}, v_{j}\right) \in \mathcal{U} \cup \mathcal{R}} x_{i j}=1 & \forall v_{i} \in \mathcal{U} \cup \mathcal{R}, \\
x_{i j} \leq x_{j} & \forall v_{j},\left(v_{i}, v_{j}\right) \in \mathcal{U} \cup \mathcal{R}, \\
\sum_{v_{j} \in \mathcal{U} \cup \mathcal{R}} x_{j}=p, & \\
x_{i j}, x_{j} \in\{0,1\} & \forall v_{j},\left(v_{i}, v_{j}\right) \in \mathcal{U} \cup \mathcal{R} .
\end{array}
$$

Therefore the sum of weighted distances of a $p$-facility location $\left\{x_{j}, x_{i j} \mid v_{j},\left(v_{i}, v_{j}\right) \in \mathcal{U} \cup \mathcal{R}\right\}$ is

$$
\sum_{v_{i} \in \mathcal{U} \cup \mathcal{R}} \sum_{\left(v_{i}, v_{j}\right) \in \mathcal{U} \cup \mathcal{R}} \eta_{i} \xi_{i j} x_{i j}
$$

which is obviously an uncertain random variable. Its chance distribution function is denoted by $\Psi(z)$, i.e.,

$$
\Psi(z)=C h\left\{\sum_{v_{i} \in \mathcal{U} \cup \mathcal{R}} \sum_{\left(v_{i}, v_{j}\right) \in \mathcal{U} \cup \mathcal{R}} \eta_{i} \xi_{i j} x_{i j} \leq z\right\} .
$$

Based on Theorem 2.2, we suggest the following algorithm to calculate the chance distribution function which corresponds to the $p$-facility location

$$
\left\{x_{j}, x_{i j} \mid v_{j},\left(v_{i}, v_{j}\right) \in \mathcal{U} \cup \mathcal{R}\right\}
$$

\section{Algorithm 2}

1. Step 1 as described in Algorithm 1.

2. For given $y_{k}$ and $y_{i j}$ and each $\alpha \in\{0.01,0.02, \ldots, 0.99\}$, calculate $F^{-1}\left(\alpha ; y_{k}, y_{i j}, v_{k},\left(v_{i}, v_{j}\right) \in \mathcal{R}\right)$, which is the sum of the weighted distances of the corresponding $p$-facility location.

3. Use discrete form of linear interpolation to obtain the uncertainty distribution function of $F\left(z ; y_{k}, y_{i j} \mid v_{k},\left(v_{i}, v_{j}\right) \in \mathcal{R}\right)$. 
A Solution Algorithm for $p$-Median Location Problem on Uncertain Random Networks 65

4. Input $F\left(z ; y_{k}, y_{i j} \mid v_{k},\left(v_{i}, v_{j}\right) \in \mathcal{R}\right)$ into formula

$$
\int_{0}^{\infty} \cdots \int_{0}^{\infty} F\left(z ; y_{k}, y_{i j} \mid v_{k},\left(v_{i}, v_{j}\right) \in \mathcal{R}\right) \prod_{v_{k} \in \mathcal{R}} d \Psi_{k}\left(y_{k}\right) \prod_{\left(v_{i}, v_{j}\right) \in \mathcal{R}} d \Psi_{i j}\left(y_{i j}\right)
$$

and calculate the chance distribution function $\Psi(z)$ for each $z$.

Theorem 4.1. Let $(\mathcal{V}, \mathcal{U}, \mathcal{R}, \mathcal{W})$ be an uncertain random network and let $\Phi(z)$ be an ideal chance distribution function associated with it. Assume that $\left\{x_{j}, x_{i j} \mid v_{j},\left(v_{i}, v_{j}\right) \in \mathcal{U} \cup \mathcal{R}\right\}$ is a given $p$-facility location. Then we have

$$
\Psi(z) \leq \Phi(z)
$$

Proof. Obviously, by Theorem 3.1, the ideal chance distribution function of the $p$-median $\Phi(z)$ is the smallest sum distribution. So we can obtain the chance distribution function of any $p$-facility location

$$
\Psi(z) \leq \Phi(z)
$$

The theorem is proved.

In order to find the $p$-median location of an uncertain random network $(\mathcal{V}, \mathcal{U}, \mathcal{R}, \mathcal{W})$, we give the following definition.

Definition 4.1. Let $(\mathcal{V}, \mathcal{U}, \mathcal{R}, \mathcal{W})$ be an uncertain random network and let $\Phi(z)$ be the ideal chance distribution function of the $p$-median. Assume that $\Psi(z)$ is the chance distribution function of a $p$-facility location $\left\{x_{j}, x_{i j} \mid v_{j},\left(v_{i}, v_{j}\right) \in \mathcal{U} \cup \mathcal{R}\right\}$. If $\Psi(z)$ is the closest the $\Phi(z)$, i.e.,

$$
\int_{0}^{\infty}\{\Phi(z)-\Psi(z)\} d z
$$

is minimum, then the $p$-facility location $\left\{x_{j}, x_{i j} \mid v_{j},\left(v_{i}, v_{j}\right) \in \mathcal{U} \cup \mathcal{R}\right\}$ is called the $p$-median in an uncertain random network.

Based on Definition 4.1 and the above statements, we formulate the following optimization model to determine a $p$-median for an uncertain random network.

$$
\begin{array}{lll}
\left(\mathbf{P}_{3}\right): \min & \int_{0}^{\infty}\{\Phi(z)-\Psi(z)\} d z & \\
\text { s.t. } & \sum_{\left(v_{i}, v_{j}\right) \in \mathcal{U} \cup \mathcal{R}} x_{i j}=1 & \forall v_{i} \in \mathcal{U} \cup \mathcal{R}, \\
& x_{i j} \leq x_{j} & \forall v_{j},\left(v_{i}, v_{j}\right) \in \mathcal{U} \cup \mathcal{R}, \\
& \sum_{v_{j} \in \mathcal{U} \cup \mathcal{R}} x_{j}=p, & \\
& x_{i j}, x_{j} \in\{0,1\} & \forall v_{j},\left(v_{i}, v_{j}\right) \in \mathcal{U} \cup \mathcal{R},
\end{array}
$$


where $\Phi(z)$ is the ideal chance distribution function and

$$
\Psi(z)=C h\left\{\sum_{v_{i} \in \mathcal{U} \cup \mathcal{R}} \sum_{\left(v_{i}, v_{j}\right) \in \mathcal{U} \cup \mathcal{R}} \eta_{i} \xi_{i j} x_{i j} \leq z\right\}
$$

is the chance distribution function of any $p$-facility location.

In order to find the $p$-median in an uncertain random network $(\mathcal{V}, \mathcal{U}, \mathcal{R}, \mathcal{W})$, we propose the following algorithm.

\section{Algorithm 3}

1. Calculate the ideal chance distribution function for the uncertain random network by using Algorithm 1.

2. Consider all the $p$-facility locations in the uncertain random network.

3. Calculate the chance distribution function of the sum of weighted distances for each $p$-facility location by using Algorithm 2 .

4. Calculate the objective function of Model $\left(\mathbf{P}_{3}\right)$ for given $p$-facility location, and choose the minimum value of objective function, which corresponds to the desired $p$-median.

\section{An illustrative example}

In this section, we give an example for the 2-median location problem in an uncertain random network to illustrate the proposed algorithms.

Consider goods distribution system. Assume that the system is given as the network $N$ in Figure 5.1 where the vertices denote urban areas. In this system, warehouses of the distribution company and supermarkets are facilities and clients, respectively. There is a supermarket at each area. Suppose that the weight $\eta_{i}$ of the vertex $v_{i}$ is equal to the average monthly purchase of residents of this area from the supermarket located at vertex $v_{i}$. Some of the vertex weights and the distances between vertices in the network are uncertain, while others are random (see Table 5.1). Our aim is to find two vertices on the network $N$ to locate warehouses of the distribution company which will minimize the sum of weighted distances from each supermarket to its closest warehouse.

$$
\begin{aligned}
& \mathcal{V}=\left\{v_{1}, v_{2}, v_{3}, v_{4}, v_{5}, v_{6}\right\}, \\
& \mathcal{U}=\left\{v_{1}, v_{2}, v_{4}, v_{5}, v_{6}\right\} \cup\left\{\left(v_{1}, v_{2}\right),\left(v_{1}, v_{4}\right),\left(v_{2}, v_{5}\right),\left(v_{3}, v_{4}\right)\left(v_{4}, v_{6}\right)\right\}, \\
& \mathcal{R}=\left\{v_{3}\right\} \cup\left\{\left(v_{2}, v_{3}\right)\right\}, \\
& \mathcal{W}=\left\{\eta_{1}, \eta_{2}, \eta_{3}, \eta_{4}, \eta_{5}, \eta_{6}\right\} \cup\left\{\xi_{12}, \xi_{14}, \xi_{23}, \xi_{25}, \xi_{34}, \xi_{46}\right\} .
\end{aligned}
$$

Note that if the distance between some vertices or weight of some vertices are considered as constant values, then we regard them as special uncertain variables. 


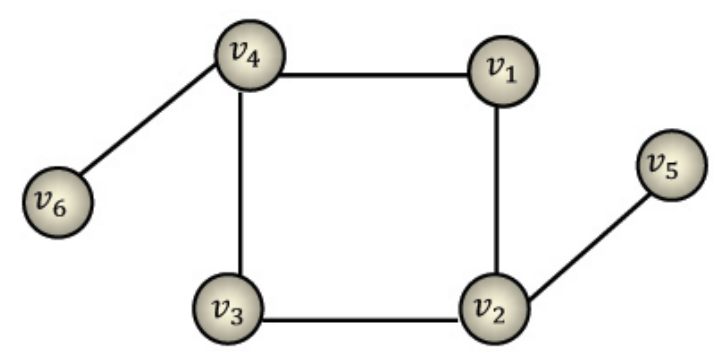

FIG. 5.1: An uncertain random network

Table 5.1: Uncertainty and probability distributions

\begin{tabular}{|c|c|c|c|}
\hline vertex & $\eta_{i}$ & arc & $\xi_{i j}$ \\
\hline$v_{1}$ & 2 & $\left(v_{1}, v_{2}\right)$ & $\mathcal{L}(2,3)$ \\
$v_{2}$ & $\mathcal{L}(2,4)$ & $\left(v_{1}, v_{4}\right)$ & $\mathcal{L}(3,4)$ \\
$v_{3}$ & $U(2,3)$ & $\left(v_{2}, v_{3}\right)$ & $U(4,6)$ \\
$v_{4}$ & 3 & $\left(v_{2}, v_{5}\right)$ & 2 \\
$v_{5}$ & $\mathcal{L}(1,2)$ & $\left(v_{3}, v_{4}\right)$ & $\mathcal{L}(2,3)$ \\
$v_{6}$ & $\mathcal{L}(2,3)$ & $\left(v_{4}, v_{6}\right)$ & 5 \\
\hline
\end{tabular}

From Theorem 3.1, we can obtain the ideal chance distribution function associated with the network $(\mathcal{V}, \mathcal{U}, \mathcal{R}, \mathcal{W})$ as follows:

$$
\Phi(z)=\int_{0}^{\infty} \int_{0}^{\infty} F\left(z ; y_{3}, y_{23}\right) d \Psi_{3}\left(y_{3}\right) d \Psi_{23}\left(y_{23}\right)
$$

where $F\left(z ; y_{3}, y_{23}\right)$ is determined by its inverse uncertainty distribution function

$F^{-1}\left(\alpha ; y_{3}, y_{23}\right)=f\left(\Upsilon_{1}^{-1}(\alpha), \Upsilon_{2}^{-1}(\alpha), y_{3}, \Upsilon_{4}^{-1}(\alpha), \Upsilon_{5}^{-1}(\alpha), \Upsilon_{6}^{-1}(\alpha) ; \Upsilon_{12}^{-1}(\alpha), \Upsilon_{14}^{-1}(\alpha), y_{23}\right.$

$$
\left.\Upsilon_{25}^{-1}(\alpha), \Upsilon_{34}^{-1}(\alpha), \Upsilon_{46}^{-1}(\alpha)\right)
$$

Give a partition $\prod_{3}$ on interval $[2,3]$ with step 0.01 , and let random variable $\eta_{3}$ take values in

$$
\left\{y_{3} \mid y_{3}=2+0.01 i \text { for } i=1, \ldots, 100\right\} \text {. }
$$

Also give a partition $\prod_{23}$ on interval $[4,6]$ with step 0.01 , and let random variable $\eta_{23}$ take values in

$$
\left\{y_{23} \mid y_{23}=4+0.01 i \text { for } i=1, \ldots, 200\right\} \text {. }
$$


For any $y_{3} \in \prod_{3}, y_{23} \in \prod_{23}$, given $\alpha \in\{0.01,0.02, \ldots, 0.99\}$, we calculate $F^{-1}\left(\alpha ; y_{3}, y_{23}\right)$ by using the model $\left(\mathbf{P}_{2}\right)$.

We may first calculate the ideal chance distribution function $\Phi(z)$ associated with the network by Algorithm 1.

Then we will calculate the chance distribution function of the total weighted distance of each 2 -facility location( i.e., $\Psi(z)$ ) by Algorithm 2, which is shown in Figure 5.2.
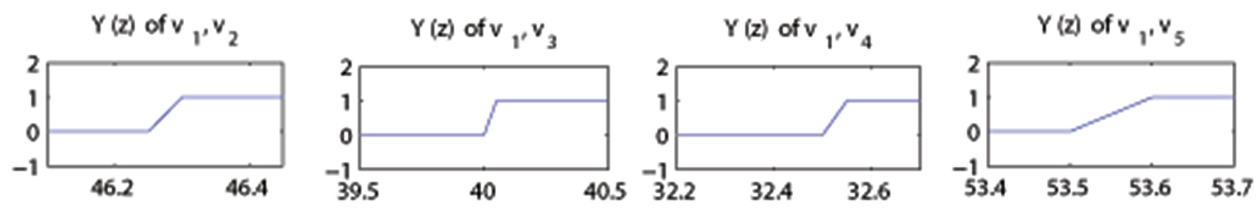

$\mathrm{Y}(\mathrm{z})$ of $\mathrm{v}_{1}, \mathrm{v}_{6}$
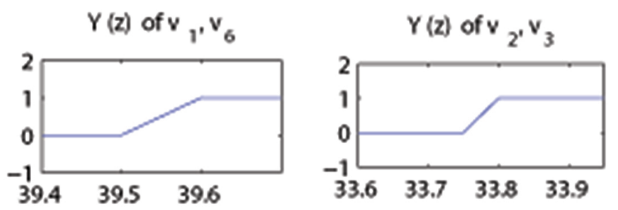

$\mathrm{Y}(\mathrm{z})$ of $\mathrm{v}_{2^{\prime}} \mathrm{v}_{4}$ (ideal)

$Y(z)$ of $v_{2}, v_{5}$
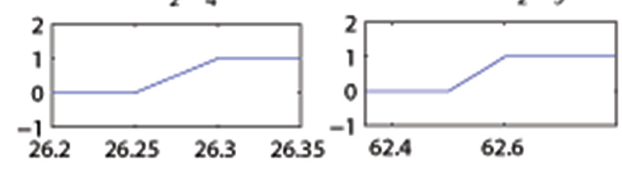

$\mathrm{Y}(\mathrm{z})$ of $\mathrm{v}_{2}, \mathrm{v}_{6}$
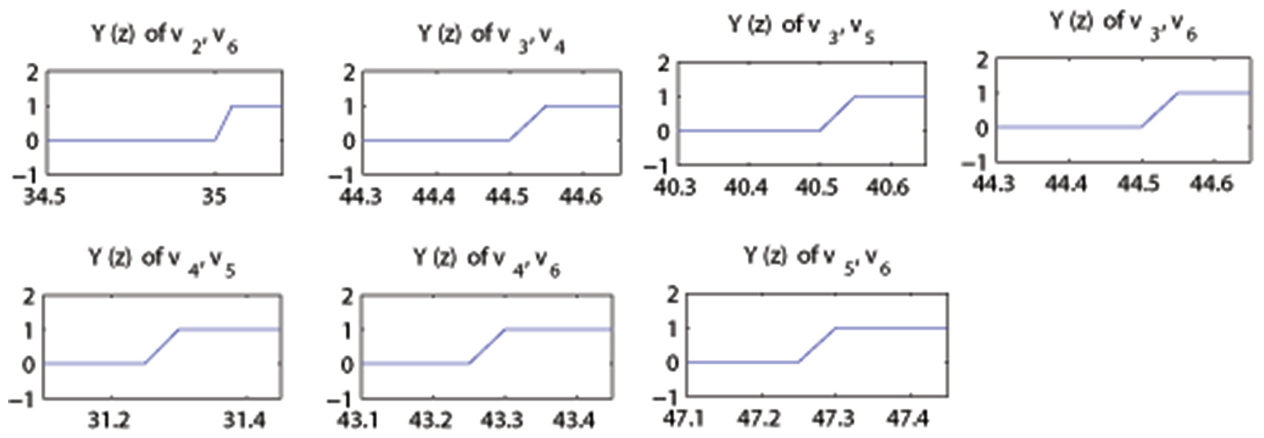

FIG. 5.2: The shapes of chance distribution functions of all 2-facilities for the example

Using Algorithm 3, we will calculate the difference between the chance distribution function of total weighted distance of each 2 -facility location and the ideal one, which is given as Table 5.2.

Model $\left(\mathbf{P}_{3}\right)$ implies that the vertices $v_{2}$ and $v_{4}$ are the desired warehouses locations since the difference between the chance distribution function and the ideal one is minimum. 
Table 5.2: Difference between the chance distribution function and the ideal chance distribution function

\begin{tabular}{|c|c|c|c|c|c|}
\hline 2-facility location & $\left\{v_{1}, v_{2}\right\}$ & $\left\{v_{1}, v_{3}\right\}$ & $\left\{v_{1}, v_{4}\right\}$ & $\left\{v_{1}, v_{5}\right\}$ & $\left\{v_{1}, v_{6}\right\}$ \\
\hline Difference & 20.015 & 13.759 & 6.259 & 27.234 & 13.234 \\
\hline 2-facility location & $\left\{v_{2}, v_{3}\right\}$ & $\left\{v_{2}, v_{4}\right\}$ & $\left\{v_{2}, v_{5}\right\}$ & $\left\{v_{2}, v_{6}\right\}$ & $\left\{v_{3}, v_{4}\right\}$ \\
\hline Difference & 7.511 & 0 & 36.234 & 8.759 & 18.261 \\
\hline 2-facility location & $\left\{v_{3}, v_{5}\right\}$ & $\left\{v_{3}, v_{6}\right\}$ & $\left\{v_{4}, v_{5}\right\}$ & $\left\{v_{4}, v_{6}\right\}$ & $\left\{v_{5}, v_{6}\right\}$ \\
\hline Difference & 14.261 & 18.261 & 5.009 & 17.009 & 21.006 \\
\hline
\end{tabular}

\section{Conclusions}

In this paper, we have investigated the $p$-median location problem in an uncertain random network, i.e., a network in which the weights of vertices and the distances between vertices are uncertain random variables. We first introduced the concept of the ideal chance distribution function and then presented an algorithm to calculate the ideal chance distribution function of the $p$-median associated with the uncertain random network. We have formulated the discrete $p$-median location problem in an uncertain random network and presented an algorithm to find the $p$-median. Finally, to illustrate the efficiency of the proposed method, we gave a numerical example.

\section{REF E R E N C E S}

1. R. Benkoczi and B. Bhattacharya: A new template for solving p-median problems for trees in sub-quadratic time (extended abstract). LNCS. 3669 (2005), 271-282.

2. O. Berman and J. WANG: Probabilistic location problems with discrete demand weights. Networks. 44 (2004), 47-57.

3. O. BERMAN and J. WANG: The network p-median problem with discrete probabilistic demand weights. Comput. Oper. Res. 37 (2010), 1455-1463.

4. O. Berman and Z. Drezner: The p-median problem under uncertainty. Eur J. Oper. Res. 189 (2008), 19-30.

5. O. BERMAN and D. KRASS: On n-facility median problem with facilities subject to failure facing uniform demand. Dis. Appl. Math. 159 (2011), 420-432.

6. S. Bespamyatnikh, B. Bhattacharya, M. Keil, D. Kirkpatrick and M. SEGAL: Efficient algorithms for centers and medians in interval and circular-arc graphs. Networks. 9 (2002), 144-152.

7. S. C. Chang, W. C. K. Yen, Y. L. Wang and J. J. Liu: The connected p-median problem on block graphs. Optim. Lett. 10 (2016), 1191-1201.

8. M. S. DASKIN: Network and discrete location : models, algorithms, and applications. 2nd ed., Department of Industrial and Operations Engineering, (2013). 
9. Z. Drezner and S. SHIODE: A distribution map for the one-median location problem on a network. Eur. J. Oper. Res. 179 (2007), 1266-1273.

10. B. GAvish and S. SRIDHAR: Computing the 2-median on tree networks in $O(n \log n)$ time. Networks. 26 (1995), 305-317.

11. H. Y. GuO and X. S. WANG: Variance of uncertain random variables. J. Uncertainty. Anal. Appl. 2 (2014), 1-7.

12. Y. GAO: Uncertain models for single facility location problems on networks. Appl. Math. Model. 36 (2012), 2592-2599.

13. S. L. HAKIMI: Optimum locations of switching centres and the absolute centres and medians of a graph. Oper. Res. 12 (1964), 450-459.

14. J. HATzL: Median problems on wheels and cactus graphs. Computing. 80 (2007), 377-393.

15. Y. C. Hou: Subadditivity of chance measure. J. Uncertainty Anal. Appl. 2 (2014), $1-9$.

16. O. KARIV and S. L. HAKIMI: An algorithmic approach to network location problem, Part 2: the p-median. SIAM J. Appl. Math. 37 (1979), 539-560.

17. A. N. Kolmogorov: Grundbegriffe der wahrscheinlichkeitsrechnung. Julius Springer, Berlin, 1933.

18. B. LiU: Uncertainty theory. 4nd ed., Springer-Verlag, Berlin, 2015.

19. B. LIU: Some research problems in uncertainty theory. 2nd ed., J. Uncertain Syst. 3 (2009), 3-10.

20. B. LiU: Why is there a need for uncertainty theory?. J. Uncertain Syst. 6 (2012), 3-10.

21. Y. H. LiU: Uncertain random variables: A mixture of uncertainty and randomness. Soft Comput. 17 (2013), 625-634.

22. Y. H. LIU: Uncertain random programming with applications. Fuzzy Optim. Decis. Mak. 12 (2013), 153-169.

23. F. V. Louveaux: Stochastic location analysis. Location Science. 125 (1993), 127-154.

24. F. V. Louveaux and D. Peeters: A dual-based procedure for stochastic facility location. Oper. Res. 40 (1992), 564-573.

25. F. V. Louveaux and J. F. Thisse: Production and location on a network under uncertainty. Oper. Res. Lett. 4 (1985), 145-149.

26. P. B. Mirchandani and A. R. Odoni: Location of medians on stochastic networks. Transp. Sci. 13 (1979), 85-97.

27. P. B. Mirchandani and A. R. Odoni: Localizing 2-medians on probabilistic and deterministic tree networks. Networks. 10 (1980), 329-350.

28. P. B. Mirchandani: The p-median problem and generalizations. Discrete location theory, Wiley-Interscience, New York, 1990.

29. K. T. NGuyen and N. T. L. Chi: A model for the inverse 1-median problem on trees under uncertain costs. Opusc. Math. 36 (2016), 513-523.

30. K. T. Nguyen, P. Van Chien, L. H. Hai and H. D. Quoc: A simple linear time algorithm for computing a 1-median on cactus graphs. Appl. Appl. Math. 12 (2017), 70-77. 
A Solution Algorithm for $p$-Median Location Problem on Uncertain Random Networks 71

31. Y. Sheng and Y. GAO: Shortest path problem of uncertain random network. Comput. Ind. Eng. 99 (2016), 97-105.

32. Y. SHEnG, Z. QIN and G. SHI: Minimum spanning tree problem of uncertain random network. J. Intell. Manuf. 28 (2017), 565-574.

33. Y. Sheng and J. GaO: Chance distribution of the maximum flow of uncertain random network. J. Uncertainty Anal. Appl. 2 (2014), 1-14.

34. Y. Sheng K. Yao: Some formulas of variance of uncertain random variable. J. Uncertainty Anal. Appl. 2 (2014), 1-10.

35. A. Soltanpour, F. Baroughi and B. Alizadeh: Classical center location problem under uncertain environment. Int. J. Ind. Math. 9 (2017), 365-374.

36. A. TAMIR: $A n O\left(p n^{2}\right)$ algorithm for the p-median and related problems on tree graphs. Oper. Res. Lett. 19 (1996), 59-64.

37. R. TAdeI, N. Ricciardi and G. Perboli: The stochastic p-median problem with unknown cost probability distribution. Oper. Res. Lett. 37 (2009), 135-141.

38. K. E. WANG and Q. YANG: Hierarchical facility location for the reverse logistics network design under uncertainty. J. Uncertain Syst. 8 (2014), 255-270.

39. M. Wen, Z. Qin, R. KANG and Y. YANG: The capacitated facility locationallocation problem under uncertain environment. J. Intell. Fuzzy Syst. 29 (2015), 2217-2226.

40. K. YAO and J. GAO: Law of large numbers for uncertain random variables. IEEE Trans. Fuzzy Syst. 24 (2016), 615-621.

Akram Soltanpour

Department of Applied Mathematics

Faculty of Basic Sciences

Sahand University of Technology, Tabriz, Iran

soltani_akram32@yahoo.com

Fahimeh Baroughi

Department of Applied Mathematics

Faculty of Basic Sciences

Sahand University of Technology, Tabriz, Iran

baroughi@sut.ac.ir

(Corresponding author)

Behrooz Alizadeh

Department of Applied Mathematics

Faculty of Basic Sciences

Sahand University of Technology, Tabriz, Iran

alizadeh@sut.ac.ir 